Notfall Rettungsmed 2018 $21: 146$ https://doi.org/10.1007/s10049-017-0397-0 Online publiziert: 14. Dezember 2017 (c) Springer Medizin Verlag GmbH, ein Teil von Springer Nature 2017

CrossMark

M. K. König · F. Flake · T. Semmel

Deutscher Berufsverband Rettungsdienst e. V., Lübeck, Deutschland

\title{
Schlaganfall - Lassen sich die Zeiten optimieren?
}

von 183 Patienten mit Notarztversorgung vs. 29 Patienten ohne Notarztversorgung.

Aus unserer Sicht zeigt sich hier, dass die Erhebung einer korrekten Arbeitsdiagnose „Verdacht auf Schlaganfall“ durch Rettungsfachpersonal genauso sichergestellt werden kann wie in einer Versorgung mit einem Notarzt. Auch zeigt sich die signifikante Verkürzung der Versorgungszeit in der Einsatzstrategie ohne Notarzt.

Die Ursachen dieses Unterschiedes in den Versorgungszeiten können wir nicht beurteilen.

Allerdings scheint es so, als ob die Anwesenheit des Notarztes bei der Übergabe die Akzeptanz der Klinikmitarbeiter gegenüber der Arbeitsdiagnose erhöht.

Die in der Versorgung von Schlaganfallpatienten eingebundenen Kliniken müssen durch Schulungen dafür sorgen, dass Anmeldungen durch Rettungsfachpersonal in gleicher Weise akzeptiert werden wie Voranmeldungen von notarztbesetzten Rettungsmitteln. Die innerklinischen Versorgungsabläufe müssen bei jeder Voranmeldung in gleicher Weise anlaufen und so zu einer schnellen innerklinischen Beurteilung und Weiterversorgung führen. Ebenfalls sollten $z$. B. durch klare rettungsdienstliche Beurteilungs- und Versorgungspfade die Patienteneinschätzung sowie die Abfrage relevanter Informationen bei Schlaganfallpatienten weiter verbessert werden. Eine standardisierte Voranmeldung kann helfen, diese Informationen mit hoher Sicherheit $\mathrm{zu}$ übermitteln.

Ähnliche Optimierungen der Versorgungskette durch Schärfung des Bewusstseins zwischen Rettungsdienst und Krankenhaus sind bei anderen Krankheitsbildern bereits gut etabliert und belegt. (z. B.
FiTTSTEMI zur Versorgung von Patienten mit ST-Hebungsinfarkt).

Der akute Schlaganfall ohne klare Bedrohung oder Einschränkung von Vitalfunktionen ist aus unserer Sicht keine Indikation zur Alarmierung eines Notarztes und muss vom auf die lokalen Gegebenheiten geschulten Rettungsfachpersonal unter besonderer Beachtung einer umfassenden Informationsgewinnung schnellstmöglich der Klinik zugeführt werden.

\section{Korrespondenzadresse}

\section{F. Flake}

Deutscher Berufsverband Rettungsdienst e.V. Maria-Goeppert-Str. 1, 23562 Lübeck,

Deutschland

flake@dbrd.de

Interessenkonflikt. M. König, F. Flake und T. Semmel geben an, dass kein Interessenkonflikt besteht. 